\title{
Human Emotion Recognition using GSR and EEG
}

\author{
*B. Tech Student, Electronics and Instrumentation Engg. \\ Narula Institute of Technology \\ Susmita Das** \\ **Assistant Professor, Electronics and Instrumentation Engg. \\ Narula Institute of Technology \\ DOI: 10.29322/IJSRP.10.05.2020.p10146 \\ http://dx.doi.org/10.29322/IJSRP.10.05.2020.p10146
}

Trisha Paul*, Chaitali Bhattacharyya*, Payosmi Sen*, Riya Prasad*, Suraj Shaw*

\begin{abstract}
Human beings are capable of expressing thousands of emotions. The complexity, intensity and involvement with that emotion also vary with the situation. Various mood disorders can be recognized by the differentiated characteristics and features. The process of analyzing human emotional state is known as emotion recognition. In this paper the main focus is on analyzing a human's emotional states in a particular period of time. So, a dimmed tendency of different psychological states are observed over a short span of time in a wide range of age groups. The main concentration is on the four major parameters such as GSR, BPM, Body Temperature, and EEG (Electroencephalogram) for detecting the emotional states. To get an accurate result GSR (Galvanic Skin Resistance) is used depending on the principle of Electro-dermal activity (EDA) which shows various electrical signal attributes with changes in human activities (which involves sweating in time of anxiousness etc.). BPM (Beats Per Minute) which acts simultaneously to give a precise heart rate count at that particular time. EEG adds up for collecting exact brain signals of the subject using electrodes revealing the psychological state. Body temperature acts as a helping hand when measured along with, as different emotions leads to the secretion of hormones which causes a slight variation in the body temperature.
\end{abstract}

Index Terms: Emotion, GSR, EEG, BPM, Temperature.

\section{INTRODUCTION}

G SR stands for Galvanic Skin Response. It is a way of measuring the electrical conductivity of the skin. Strong emotions such as anxiety, fear, stress and cheerfulness can create a stimulus in the human nervous system, resulting in more sweat being sent to the skin surface. Electro dermal activity (EDA) is a property of the human body that causes continuous variations in the electrical processes of the skin after exposure. The activity of the sweat glands is caused by the postganglionic sudomotor fibers. [1]. The most common measure of a GSR signal is not resistance, but conductance. Conductance is the electrical opposite phenomena of resistance and is measured in Siemens unit $(C=1 / r)$. The conductance makes the signal interpretation easier, since the greater the sweat gland activity, the higher the skin conductance. The most common method to measure a GSR signal for emotional research purposes is based on a constant voltage system (exosomatic method). The GSR sensor applies a constant voltage usually $0.5 \mathrm{~V}$ to the two electrodes placed in contact with the skin. To analyse the emotional state the BPM (Beats Per Minute) parameter variations in the subject's body must be observed. Experts say that an adult human being has 60-100 beats per minute and 60-85 beats is a standard BPM range for a normal human body. In stressed condition, the BPM rate increases to around 38 beats more. BPM measurement is quite helpful to analyse stressed condition or emotional state. Temperature is another important parameter because in stressed condition the secretion of Adrenaline hormone creates some changes in human body to face the condition with the change in body temperature. B.P. i.e. Blood Pressure changes when the body temperature is increased as a result of Adrenaline secretion, then blood flow is increased specially in the capillaries under the skin to produce sweat quickly to release body heat. A normal human being has standard systolic pressure in between $90-120 \mathrm{mmHg}$ and standard diastolic pressure in between $60-80 \mathrm{mmHg}$. When a human is in stress or scared or in any other emotional state then there changes occur in the blood pressure.

EEG (Electroencephalogram) is a method in which human brain electrical activity in any emotional state can be recorded and monitored. EEG signals are mainly non-stationery and time varying in nature. EEG consists of waves such as Delta wave (0.1-4 Hz), Alpha wave $(4-8 \mathrm{~Hz})$, Theta wave $(8-13 \mathrm{~Hz})$, Beta $(13-30 \mathrm{~Hz})$, Gamma $(30-40 \mathrm{~Hz})$. These brain waves are usually sinusoidal in nature and are measured from peak to peak which is normally ranged from 0.5 to $100 \mu \mathrm{V}$. Mainly Alpha wave is responsible for stress due to tension and excitement [2]. Emotion can be intentionally controlled where EEG or biometric signal is used for better result. EEG and GSR change in a specific way when human face certain situations [3]. In response to the Automatic Nervous System (ANS), the physiological signals 
change in according to Connon's theory. The current method is better than the other methods as the CNS (Central Nervous System) and ANS are largely reflexively active and hence, cannot be easily controlled. [4] In the work of [5] the authors have used GSR as one of the inputs to recognize five emotions. Six emotions were classified from GSR signals by using Fisher Classifier. 30 statistical features have been extracted including maximum and minimum values of GSR [6]. The average verifying recognition rates of surprise (78\%), fear (73\%), disgust (70\%), grief (62\%), happy (62\%) and angry or stress (45\%). This paper [7] discussed about using EEG as an input for emotion detection through multidimensional information in EMD (Electro Mechanical Dissociation) domain. In the work of [8] the authors have used GSR as one of the inputs to recognize five emotions. Six emotions were classified from GSR signals by using Fisher Classifier. 30 statistical features have been extracted including maximum and minimum value of GSR [9]. The average verifying recognition rates of surprise (78\%), fear (73\%), disgust (70\%), grief (62\%), happy (62\%) and angry or stress (45\%). This paper [10] discussed about using EEG as an input for emotion detection through Multidimensional Information in EMD Domain.

\section{METHODOLOGY}

There are some steps to be followed to achieve the desired emotional state recognition using GSR and EEG.

\section{A. Data Acquisition:}

Data acquisition is needed to collect information about the physical parameters. For this case, GSR electrodes are placed at palms, fingers where at the other side the EEG electrodes are placed on the scalp according to international 10-20 scalp electrode placement system. [11] The transducer device will convert the physical parameters into the electrical signal. Signal needs to be filtered and amplified. Once the signal is digitized, the signal is encoded to reduce and correct transmission errors. [12]



Figure 1: Block Diagram of Emotion Recognition workflow using GSR and EEG

\section{B. Feature Extraction \& Selection:}

Feature extraction methods include modified and fixed custom symbols and structure, structure definitions and graphs. Feature selection is about reducing dimension and it causes classification accuracy [13]. It is also called variable selection, attribute selection or variable subset selection [14].

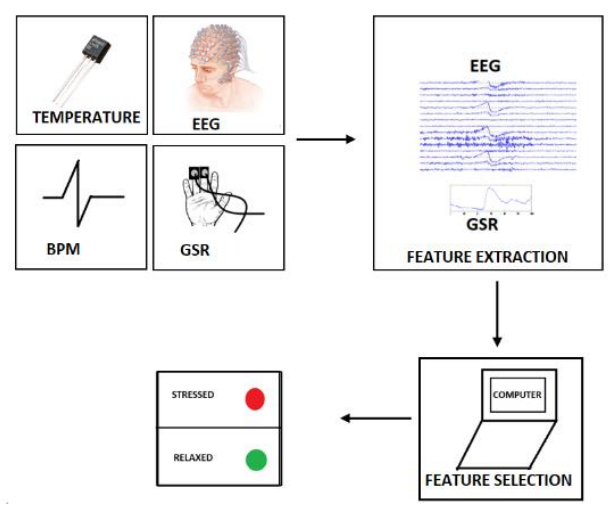


Figure 2: Schematic Diagram of Stressed and Relaxed stage detection

C. Workflow:

After extracting and selecting features the features are to be differentiated between two separate cases. For the current research work, for the stressed and relaxed situation detection, the workflow is given below.

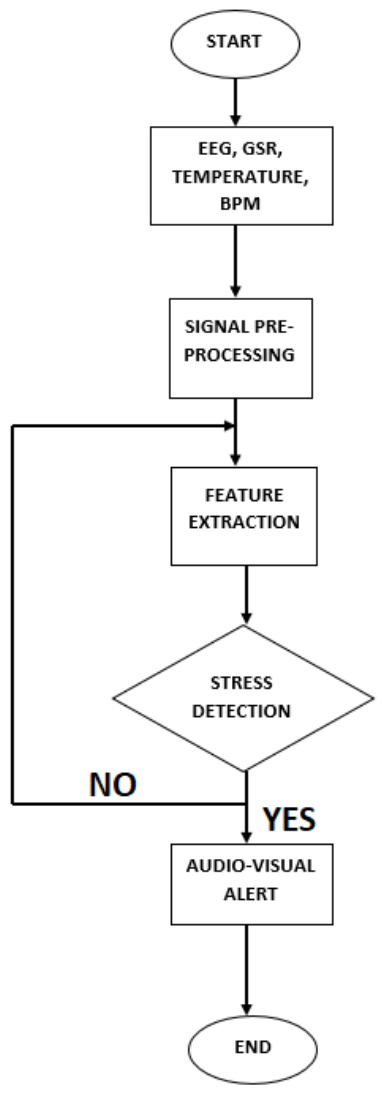

Figure 3: Flow Chart of Emotion detection

When a human brain is in stressed situation, the temperature of the body rises and then the output gives signal to the eccrine glands to conceal sweat. The sweat glands are most in numbers in the palm area and the electrical activity in brain changes as well. The electrodes must be placed on the scalp properly. For GSR silver electrodes can be used. Initially the body parameters are set as input, while the subject is in stressed situation, the physiological parameters are triggered and the body starts sweating. After acquiring the raw data, they are being filtered, pre-processed and the noise or any kind of disturbances in the signal are to be performed. Then by Arduino interfacing board the subject is tested.

\section{RESULTS AND DISCUSSIONS}

In this recent work, the experiment has been performed upon 16 users where 8 were women and 8 were men. Their age limit is 23 to 56 . The sampling rate of the signals is $4 \mathrm{~Hz}[15]$. 
Table1: ANOVA analysis of the three types of emotions

\begin{tabular}{|c|c|c|c|c|c|c|c|}
\hline $\begin{array}{l}\text { Name of } \\
\text { the } \\
\text { Situations }\end{array}$ & $\begin{array}{c}\text { Sum of } \\
\text { Squares } \\
\text { (SS) } \\
\text { (Model) }\end{array}$ & $\begin{array}{c}\text { Sum of } \\
\text { Squares } \\
\text { (SS) } \\
\text { (Error) }\end{array}$ & $\begin{array}{c}\text { Sum of } \\
\text { Squares } \\
\text { (SS) } \\
\text { (Total) }\end{array}$ & $\begin{array}{c}\text { Mean Square } \\
(\text { MS) } \\
\text { [SS(Model)/ } \\
(\mathbf{m}-1)]\end{array}$ & $\begin{array}{c}\text { Mean Square } \\
\text { Error (MSE) } \\
\text { [SS(Error)/ } \\
(\text { n-m)] }\end{array}$ & $\begin{array}{c}\text { F Value } \\
=(\mathrm{MS} / \mathrm{MSE})\end{array}$ & Prob $>F$ \\
\hline Relax & 0.10457 & 0.37842 & 0.48299 & 0.02258 & 0.0473 & 1.10527 & 0.37685 \\
\hline Operations & 0.24929 & 0.44242 & 0.69171 & 0.12464 & 0.0553 & 2.25383 & 0.16736 \\
\hline Reading & 0.21019 & 0.46367 & 0.67386 & 0.1051 & 0.05796 & 1.81328 & 0.22416 \\
\hline
\end{tabular}

ANOVA analysis means the variance analysis [16] of the three types of the mental states using acquired database in the Table 1. It is a statistical technique which is used in this recent work to compare the three emotional situation parameters of mental state. ANOVA is used to predict the mental state by observing the statistical values such as mean value difference from one emotional parameter to another. From the above table some of the features such as Sum of Squares (SS), Mean Square (MS), Mean Square Error (MSE), F Value and prob $>\mathrm{F}$ are achieved using Origin software. Sum of Squares (SS) is the addition of each Squared Deviation (SD) of the parameters. SS is calculated by a formula, $\mathrm{SS}=\sum \mathrm{x}^{2}-\left\{\left(\sum \mathrm{X}\right)^{2} / \mathrm{N}\right\}$ [where, $\mathrm{N}$ is the total number of data; $\left(\sum \mathrm{X}\right)$ is summation of total average or mean values and then calculate the square of total mean value i.e. $\left(\sum X\right)^{2} ; \sum x^{2}$ is the summation of square value individual data]. When the subjects are in relax mood, the total SS value is 0.48299 , in operations situation SS is 0.69171 and for reading time the SS value is 0.67386 . Degree of Freedom is also calculated by three steps. As total numbers of data is N, three types of situation parameters are used i.e. $\mathrm{m}$, so Degree of Freedom $\left(\mathrm{DF}_{\text {Model }}\right)=(\mathrm{m}-1)=(3-1)=2$ and Error of $\mathrm{DF}=(\mathrm{N}-\mathrm{m})$ get $\mathrm{MS}=(11-3)=8$. These $\mathrm{DF}$ values are same for rest of the parameters. Mean Square (MS) is the division of (SS Modal) by $(\mathrm{DF}$ Model $)$ i.e. MS Model $=\{\mathrm{SS} /(\mathrm{m}-1)\}$. In this present work, using this formula MS values are achieved respectively in relax, operations and reading situations i.e. 0.02258, 0.12464 and 0.1051. Mean Square Error (MSE) has been calculated same as MS Model i.e. MSE or $\mathrm{MS}_{\text {Error }}=\{\mathrm{SS} /(\mathrm{N}-\mathrm{m})\}$ and the values $0.0473,0.0553$ and 0.05796 respectively. The $\mathrm{F}$ value means the ratio between to mean square values which depends on null hypothesis [17]. $\mathrm{F}$ value is calculated by using a formula i.e. $\mathrm{F}=\left(\mathrm{MS}_{\text {Model }} / \mathrm{MSE}\right.$ or $\left.\mathrm{MS}_{\text {Error }}\right)$ and the values achieved respectively 1.10527, 2.25383 and 1.81328 in relax, operations and reading situations. Relax and reading situations show true null hypothesis value because in these two situations the $\mathrm{F}$ value are near to 1 but in the operations situation the $\mathrm{F}$ value $>1$. This null hypothesis depends on the $\mathrm{P}$ value or Probability value of $\mathrm{F}$. If the $\mathrm{P}<0.05$ (a common alpha level value for the test), then this null hypothesis can be rejected [18]. But in this recent work, the calculated Prob $>F$ values are $0.37685,0.16736$ and 0.22416 where the $P>0.05$. So that these $F$ values and Prob $>F$ values are represented statistical significance. This type of analysis is required to show the behaviour or nature of the system chosen for the experiment. Through the predictive system analysis the difference between several emotional states can be performed.

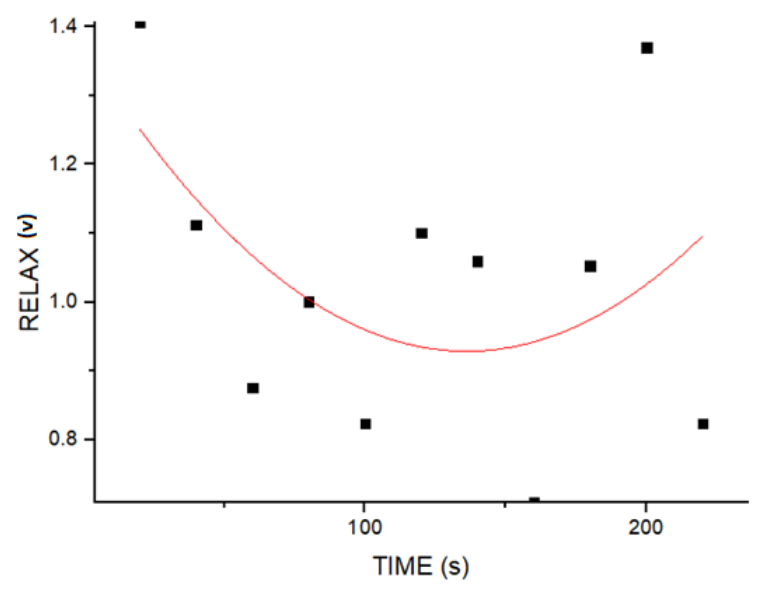

Fig.4 (b). Graphical representation of relax situation

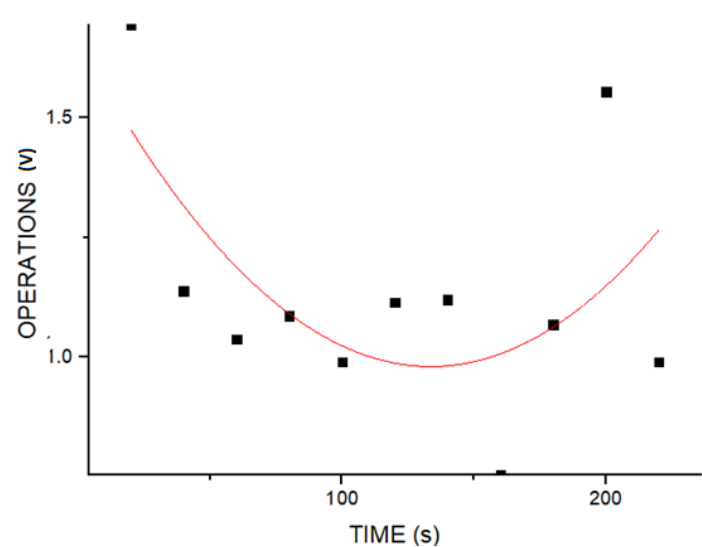

Fig.4 (b). Graphical representation of operations situation 


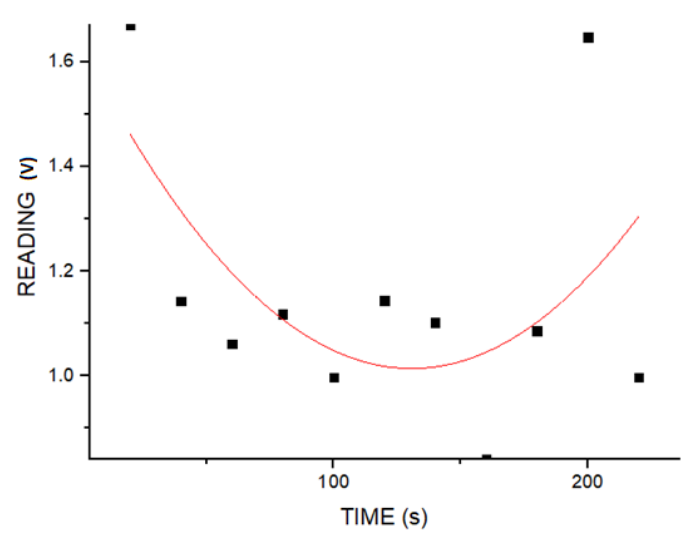

Fig.4 (c). Graphical representation of reading situation

Fig.4. Graphical representations of Fitting Plots of Relaxed, Operations and Reading situations

Fig.4 overall shows the Fitting plots of Relaxed, Operations and Reading situations (mV) in Mental State with respect to the Time in second. In the recent work, the Fitting plots are used to indicate the non-linearity, unequal error variance and outliers between these three mental states to recognize the emotional variations. Fig.4 (a) presents the variation of the output voltage $(\mathrm{mV})$ when the subjects are in relaxed situation. Fig.4 (b) represents the mental effort while the subjects are doing mathematical operations. Fig.4 (c) shows the output voltage variation when the subjects are in reading mode. In the Fig.4 (a) it has been observed that the output voltage of the average values has decreased. All the mental state situations represent a difference variations in the graph. By observing all the graphs it can be observed that the mental state varies in different mental situations.



Fig.5 Graphical representation of Mental state of RELAX and NERVOUS Situations with respect to Time

In the Fig.5 the Bar plot is used to represent the categorical data such as relax and nervous situations of mental state with rectangular bars with heights or lengths with respect to the time in second. In this case, the subjects had to think something which makes the nervous situations in their mind. For this experiment the men and women subjects are used to acquire the data in both the situations such as relax and nervous. Then the difference of the output voltage variance has been observed after observing the Bar plot. Fig.5 shows the variation difference between the two mental state situations of nervous and relax. After recording all these tested values, a prototype can be 
generated to indicate lie or true words detector using GSR sensor and EEG recorder. The emotion statement can also be declared by observing all of these graphical representations in this work.

\section{CONCLUSIONS}

The main purpose of this present work is to recognize the emotions of human being such as anxiety, nervousness and happiness, sadness. There are many processes to make these tests but in this paper the functional and non-specific properties of the skin are considered. Electrodermal activity (EDA) Grove and GSR which allows one to see such strong feelings by simply tapping two electrodes on two fingers on. To develop this system an algorithm has been used that would detect falsehoods. 16 subjects ( 8 male, 8 female) were used to collect the output voltages in different situations. The three types of situations such as relaxed, operations and reading were observed. To show the variance of the output graphical representations are used. It has been tried to develop the algorithm to establish the work more reliable. More parameters related to mental state situations help to get more accuracy in the work. The proper discrimination of the emotions depending on genders also can be a great extension of this work. Nowadays Polygraph test or lie detection test are the common useable test especially in the crime department. The Galvanic Skin Response (GSR) sensor can be used along with other parameter incorporation for the investigation of the effectiveness of using a polygraph, the Modified Comparison Questions Technique (CQT) and Beats per Minute (BPM). This test can be used to detect fraudulent passengers by carrying illegal substances in their luggage in anywhere. Polygraph tests or lie detection tests are performed using a GSR sensor and Electrodermal activities (EDRs).This polygraph or lie detection tests also can be used as an Emotion-related projects such as sleep quality monitoring. Polygraph testing process are basically applied in the investigation field. These records are used to make any device for polygraph tests or lie detection tests. The GSR devices are used in different difficult situations from being in relaxed conditions with a success rate of $90.01 \%$.

\section{ACKNOWLEDGMENT}

The authors are grateful for the support of Narula Institute of Technology.

\section{REFERENCES}

[1] Innervation territories of single sympathetic C fibers in human skin. Schmelz M, Schmidt R, Bickel A, Torebjörk HE, Handwerker HO J Neurophysiol. 1998 Apr; 79(4):1653-60.

[2] Chandra S, Jaiswal AK, Singh R, Jha D, Mittal AP. Mental Stress: Neurophysiology and Its Regulation by Sudarshan Kriya Yoga. Int J Yoga. 2017;10(2):67-72. doi:10.4103/0973-6131.205508

[3] Shu L, Xie J, Yang M, et al. A Review of Emotion Recognition Using Physiological Signals. Sensors (Basel). 2018;18(7):2074. Published 2018 Jun 28. doi:10.3390/s18072074

[4] Cannon W.B. The James-Lange theory of emotions: A critical examination and an alternative theory. Am. J. Psychol. 1927;39:106-124. doi: 10.2307/1415404.

[5] Wen W., Liu G., Cheng N., Wei J., Shangguan P., Huang W. Emotion Recognition Based on Multi- Variant Correlation of Physiological Signals. IEEE Trans. Affect. Comput. 2014;5:126-140. doi:10.1109/TAFFC.2014.2327617

[6] Wu G., Liu G., Hao M. The Analysis of Emotion Recognition from GSR Based on PSO; Proceedings of the International Symposium on Intelligence Information Processing and Trusted Computing; Huanggang, China. 28-29 October 2010; pp. 360363.

[7] Ning Zhuang, Ying Zeng, Li Tong, Chi Zhang, Hanming Zhang, and Bin Yan, Emotion Recognition from EEG Signals Using Multidimensional Information in EMD Domain.

[8] Wen W., Liu G., Cheng N., Wei J., Shangguan P., Huang W. Emotion Recognition Based on Multi-Variant Correlation of Physiological Signals. IEEE Trans. Affect. Comput. 2014;5:126-140. doi: 10.1109/TAFFC.2014.2327617

[9] Wu G., Liu G., Hao M. The Analysis of Emotion Recognition from GSR Based on PSO; Proceedings of the International Symposium on Intelligence Information Processing and Trusted Computing; Huanggang, China. 28-29 October 2010; pp. 360363. 
[10] Ning Zhuang, Ying Zeng, Li Tong, Chi Zhang, Hanming Zhang, and Bin Yan, Emotion Recognition from EEG Signals Using Multidimensional Information in EMD Domain.

[11] From Oostenveld and Praamstra, Clinical Neurophysiology, 112, 2001, 713-719

[12] Simon S. Young (2001). Computerized Data Acquisition and Analysis for the Life Sciences. Cambridge University Press. ISBN 978-0-521-56570-7.

[13] Anke Meyer-Baese, Volker Schmid, in Pattern Recognition and Signal Analysis in Medical Imaging (Second Edition), 2014

[14] Guyon, Isabelle; Elisseeff, Andre (2003). "An Introduction to Variable and Feature Selection". Journal of Machine Learning Research. 3: 1157-1182.

[15] Maria Viqueira Villarejo, Begona Garcia Zapirain and Amaia Mendez Zorrilla. A stress sensor based on Galvanic Skin Response (GSR) controlled by ZigBee.

[16] Gurchetan Singh. A Simple Introduction to ANOVA (with applications in Excel). January 15, 2018, Analytics Vidhya.

[17] James Jones, richland.edu./james/lecture/m170/ch13-1wy.

[18] Thomas J. Archdeacon (1994). Correlations and Regression Analysis. A Historian's Guide. The University of Wisconsin Press.

\section{AUTHORS}

First Author - Trisha Paul, B. Tech Student, Electronics and Instrumentation Engg., Narula Institute of Technology and trishapaul2017@gmail.com.

Second Author - Chaitali Bhattacharyya, B. Tech Student, Electronics and Instrumentation Engg, Narula Institute of Technology and chaitali1931@gmail.com.

Third Author - Payosmi Sen, B. Tech Student, Electronics and Instrumentation Engg., Narula Institute of Technology and payosmisen@gmail.com.

Forth Author - Riya Prasad, B. Tech Student, Electronics and Instrumentation Engg., Narula Institute of Technology and riyaprasad862@gmail.com.

Fifth Author - Suraj Shaw, B. Tech Student, Electronics and Instrumentation Engg., Narula Institute of Technology and surajbaiju291@gmail.com.

Sixth Author - Susmita Das, Assistant Professor, Electronics and Instrumentation Engg., Narula Institute of Technology and susmitad2011@gmail.com.

Correspondence Author - Susmita Das, Assistant Professor, Electronics and Instrumentation Engg., Narula Institute of Technology and susmitad2011@gmail.com, 8017205403. 